\title{
Developing DA Applications in SMEs Industrial Context
}

\author{
Giorgio Colombo ${ }^{1}$, Dante Pugliese ${ }^{1}$, and Caterina Rizzi ${ }^{2}$ \\ ${ }^{1}$ Politecnico di Milano, Italy, giorgio.colombo@polimi.it \\ ${ }^{2}$ Università Degli Studi di Bergamo, Italy, caterina.rizzi@unibg.it
}

\begin{abstract}
This paper discusses aspects related to the implementation of Design Automation applications within Small Medium Enterprises (SMEs) industrial context. It focuses the attention on some characteristics of the design process in SME context, and highlights issues of DA in relation to the characteristics previously evidenced. On this basis, it has been defined a methodology, named MEDEA (Methodology per Design Automation), to develop DA applications; it proposes a step by step roadmap and suggests methods and tools finalized to developers more skilled on products and design process than on IT technologies. Two industrial applications realized to evaluate the methodology are then presented. They are based on two different approaches: the first suitable to represent product structure and derived from Object Oriented programming and the second based on design process representation.
\end{abstract}

Keywords: Design Automation, Knowledge-based Engineering, SMEs, Knowledge representation, PDM/PLM

\section{Introduction}

In this paper we discuss aspects related to the implementation of Design Automation (DA) in industrial context, in particular within Small Medium Enterprises (SMEs) domain. This interest is justified by two considerations: first, we consider DA an important tool to effectively innovate and improve design procedures and, secondly, SMEs are the most interesting domain for this type of methodology, particularly in Europe where they constitute the main productive tissue.

DA can be defined as a set of methods, tools and applications that permit to automate the design process; it can be applied to all the phases of the process, from the conceptual to final one related to the production of technical documentation. However, in our opinion, it well fits the detailed design phase during which a technical solution is completely formalized. In particular, main

Please use the following format when citing this chapter:

Colombo, G., Pugliese, D. and Rizzi, C., 2008, in IFIP International Federation for Information Processing, Volume 277; Computer-Aided Innovation (CAI); Gaetano Cascini; (Boston: Springer), pp. 69-82. 
benefits from a DA application can be achieved when dealing with products and parts characterized by a well defined architecture and design process. Some examples of products falling within this category are heat exchangers, gears, machine tools and so on.

DA history matches with IT one; first applications were developed with general purpose programming languages and concerned specific aspects of design process such as kinematic analyses and synthesis. A remarkable improvement was gained when CAD techniques was developed. The integration between CAD models and programming languages permitted the development of automatic procedure to configure parts and simple products [1]. This approach was enhanced by the development of parametric CAD [2]; a lot of applications to configure parts and products have been developed by using parametric models and programmable tools such as spreadsheet. In SMEs this is actually one of more diffuse method to develop simple DA applications. The described DA evolution is "design oriented" and not focused on IT.

In fact, at the same time, a number of methods and tools derived from Artificial Intelligence appeared; in particular, Knowledge Based Engineering (KBE) tools based on Object-Oriented approach constitute the best approach to DA. They had a relevant impact within aeronautical and automotive companies [3] [4]. Side by side, researches on methods to acquire and formalize knowledge have been started. Knowledge formalization is a fundamental issue, and several efforts [5] have been carried out in this direction. KBE tools evolved from initial elementary implementation to the actual one characterized by programming language, powerful tools to define customized GUI and to integrate external programs (CAD systems, FE solvers, spreadsheet, data base, etc.).

Today KBE approach is the best solution to implement DA in industrial context; however its exploitation has not achieved relevant results, especially in SMEs. This is mainly due to resources and skills required to implement such a methodology.

In this paper we discuss the topics that, in our opinion, will permit a larger diffusion of DA in industrial context. We first introduce main issues related to the applications of DA concepts within SMEs domain, then a methodology, named MEDEA (MEthodology for DEsign Automation), specifically targeted to SMEs is presented as well as two case studies realized for its validation.

\section{DA Issues within SMEs Industrial Context}

In literature we can find various research works on DA methodologies [5-9] and applications [4] [10-11]. However most of them concerns big enterprises, such as automotive or aeronautical companies, characterized by a high number of skilled people and HW/SW tools, only few applications SMEs [7][11]. We adapted DA 
approaches to fulfill SMEs needs developing a targeted roadmap to implement KBE applications for such a context.

First, we carried out an analysis of the design processes in some SMEs to identify design situations and issues related to DA. The development of a new product is the result of two distinct sequences of activities: the first one dedicated to define and detail the product architecture and the second finalised to dimension system and parts, to choice components, to verify functional requirements. We identified two typical situations. The first one focuses on the definition of the product architecture; a family of press brakes for sheet metal bending is a typical example. The design process is centred on machine architecture and based on simple modifications of parts geometry; complex calculations are not required and some choices are based on company know-how.

In the second case the emphasis is on the design process. An example is the design of a shell-and-tube heat exchanger. The design process of such a machine starts from preliminary thermal dimensioning, continues with conceptual mechanical design and ends with detail one [12]. This articulated process requires dimensioning, standard verifications, iterations, tasks very time consuming, up to few dozen hours.

From this analysis we identify following requirements for a methodology applicable to SMEs context:

- capacity to structure product and process knowledge optimising company's design processes;

- maximize the reuse and sharing of company knowledge [13];

- integrate systems and documents within an application for product automatic configuration.

This means that a DA methodology has to deals with formalization and representation of product/process knowledge and product data management. Thus it necessary to identify and/or define proper methods and tools able to deal with above mentioned issues and affordable also by people with non specific skills on design automation, as typically happens within SMEs.

\subsection{Product Knowledge Formalization}

Several methods and tools are available in literature to represent knowledge necessary to develop a new product. They permit to formalize knowledge at different levels, capturing different aspects of the knowledge itself. In an approach based on CAD experience, the representation of product architecture is the first aspect to be considered. Gorti, S. R. et al. [14] Colombo, G. et al. [15-16] indicate Object - Oriented (O-O) approach as the most suitable and adopted technique to represent the product architecture, described usually as a tree. It approach permits to represent with a logic order the functional structure of the product and allows easily modifying, adding or deleting parts and subparts without having to heavily modify all the code. Moreover, each part or component can be 
considered independent each other; therefore, it is possible to consider them as small application, able to be applied to different levels of a tree structure of the same or other products, inheriting parents' characteristics.

To represent product knowledge, we propose to adopt UML Static Class Diagram (http://www.omg.org/uml). This type of diagrams represents the components and functional sub-assembly as O-O classes with associated properties and methods. It is possible to define relationships among properties of different classes, specifying the definition of the corresponding methods.

\subsection{Process Knowledge Formalization}

Typically, the development of a DA application requires a deep analysis of the design process, i.e., the acquisition of the knowledge the technical staff uses to design the considered product. This activity is particularly important for SMEs because the implementation of a DA application often requires a re-engineering activity to optimise the process itself and integrate company best practices with engineering knowledge. Business process modeling techniques, such as ARIS [17], IDEF (www.idef.com), UML (www.omg.org/uml) [18], can be used. There isn't a universal tool; the challenge is to find the right tool for the considered problem. We propose to adopt IDEF0 and IDEF 3 Process Flow (PF), even if more recent techniques, such as UML Eriksson-Penker Business Extension [18], have been developed.

Thanks to their graphic languages, IDEF0 and IDEF3 models can be easy understood and used also by people without a specific scientific background as sometimes happens within SMEs, thus facilitating the communication among work teams with different competences. This is particularly important to validate the model with process experts and to ensure that collected information has been correctly formalized in order to implement an adequate KBE application.

\subsection{Knowledge Re-use and Sharing}

This issue is particularly crucial in developing DA applications. Sainter [19] states that "the concept of project knowledge reuse and sharing is where the product knowledge can be shared within the same knowledge domain, but at different locations and allows the domain knowledge to be reused in new situations". Cheung [20] states that Knowledge Reuse is the adaptation of explicit knowledge (domain and/or strategic knowledge well formalized to be represented) of successful practices so as to generate new and useful ideas. From these definitions, we can ask ourselves what type of knowledge we can store to share and reuse. In a $\mathrm{KBE}$ application one can identify knowledge referred to a single part, to a product structure and to a design procedure. 
The first type is referred to the elementary and structured "pills" of knowledge involved into product design; it concerns basic components that cannot be further subdivided. For example, the knowledge involved in the choice of a screw includes the rules for sizing the screw, the geometry to create it, the document to refer to, the procedure to configure the specific item (as M10 rather than M8X1.5). This pill is considered as undividable; the same approach can be extended to all the standard parts, some specific ones and sub-assemblies that can be used in different products or contexts. Number and types of the elements stored in the knowledge base depend on the KBE applications one intends to develop.

\subsection{Integration between PDM/PLM and KBE applications}

Another important aspect in the automation of the design process is the control on the definition of new parts; in fact, a KBE application must use standard or existing parts as much as possible. We think that the solution to this problem does not to rely on the definition of a local database for the specific KBE application but it should be based on the integration of the database managed by PLM/PDM system. We propose an approach that consists in defining specific coding of stored parts to make possible their automatic identification and selection. In particular, the code should represent a set of information on the specific component; i.e., the code should give information about geometry and other aspects such as assembly procedures, adaptability to a specific use, etc... A similar approach was used also in Group Technology to identify families of parts. We know that there are some problems to generalize this approach, but the present research wants to be only a first step in this direction.

\section{MEDEA: A Roadmap for KBE Applications}

On the basis of mentioned issues and our experience in this field we defined MEDEA methodology that proposes a step by step roadmap to develop KBE applications specifically targeted to SMEs. It is characterized by a reengineering activity of the design process and the use of tools more suitable to engineers than to IT experts. The possible process reengineering is related to the fact that often small enterprises have high competencies on strategic knowledge but not in domain one (standards, calculations and so on) and the development of DA application requires first integration in this direction.

At high level, the methodology is based on four main steps:

- Specs definition: identification of DA application specs and the criteria to make re-usable and sharable blocks of the product and process knowledge;

- Knowledge acquisition: collection of the knowledge related to the product architecture, the design process and the definition of the integration 
strategies between the DA application and the data/document management system, such as a PDM (Product Data Management) system;

- Knowledge formalization: representation of product architecture (tree diagram and UML class diagram) and of the process model (IDEF0 and IDEF3 diagrams);

- Integration with PDM/PLM system: definition of the interactions among DA application, PDM system and end-users whose representation can be done using UML activity diagram;

- Implementation of DA application using a KBE system.

The choice of the KBE tools to implement the DA application depends on the considered domain. Depending on the KBE system, some activities of MEDEA cannot be carried out. In fact, some KBEs are based on process representation, others on product representation or even on a mixed product-process representation. In any case, the proposed methodology remains unchanged.

In collaboration with some Italian SMEs, MEDEA has been validated with various study cases: a shell-and-tube heat exchanger, a press brake for metal sheet, a family of industrial mixers, a spring coiling machine, and a gear box.

In the following we describe two applications: one for sheet metal brake press centered on product architecture and another one for gear box focused on process representation and integration with PDM system.

\section{4. $1^{\text {st }}$ Case Study: Sheet Metal Brake Press}

This case study concerns the automation of the design process for a hydraulic press brake family characterised by a bending force up to 250 tons and bending length up to 5 meters. It has been carried out in collaboration COLGAR Spa, an Italian SME.

This is a typical example of product for which the designer selects a configuration on the basis of few parameters (e.g., bending force) and proceeds modifying parts sizes according to customers requirements.

In this case product knowledge has been acquired and formalised according to MEDEA. The product tree structure and UML static diagrams have been developed to describe relationships among parts and properties as well as design rules.

Figure 1 shows the tree structure where one can note the subdivision of the machine into three main functional sub-systems: bending group, frame and accessories \& security. Figure 2 portrays a snapshot of the UML static class diagram representing the bending sub-system. 


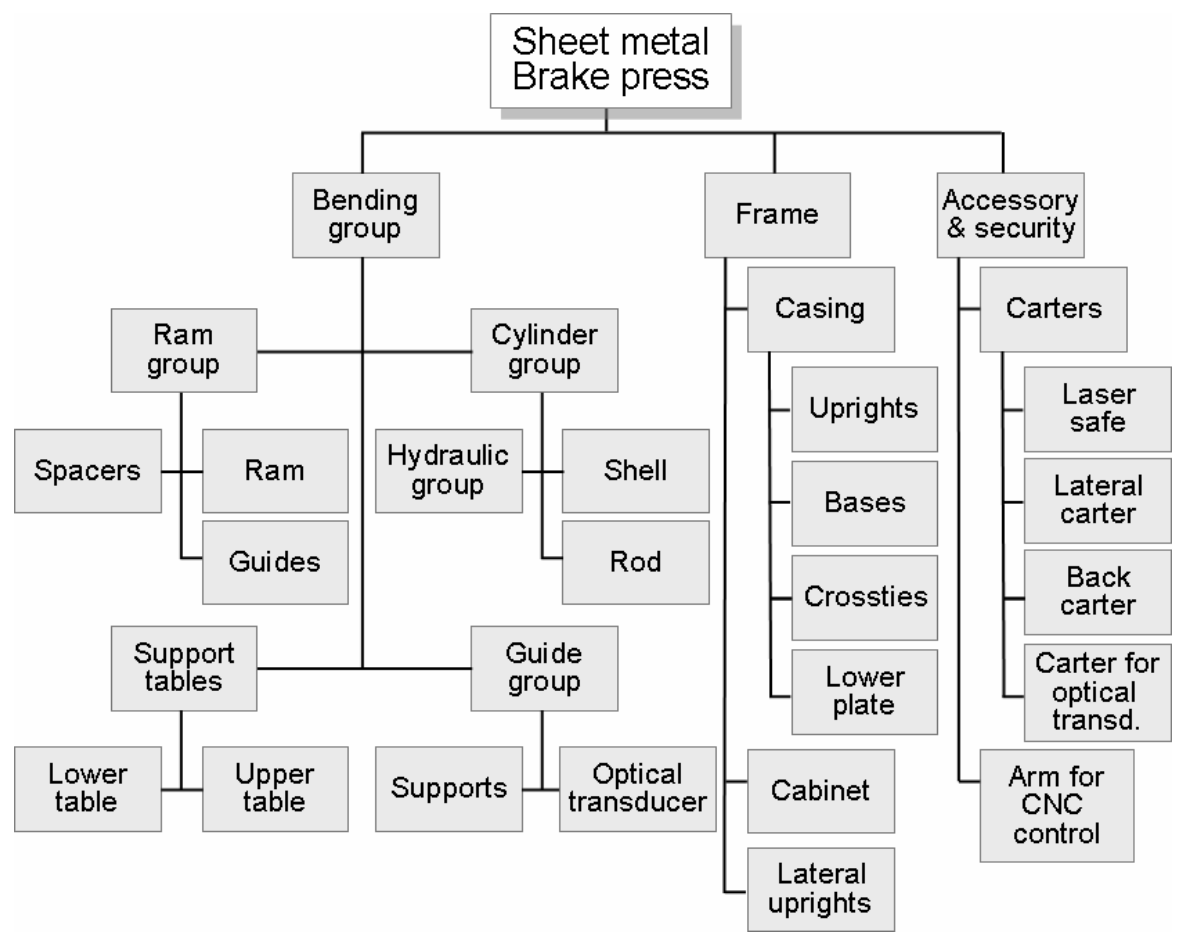

Figure 1 Tree structure of the press brake family.

The development of the DA application required the following tasks:

- definition of the press brake tree structure;

- definition of the properties of each part, sub-assemblies and complete assembly;

- rules representation;

- generation of parts and material databases;

- creation of the parametric geometric model of each part;

- automatic procedure definition;

- development of the graphical user interface.

Figure 3 shows a partial view of the tree structure implemented where each part, subassembly and assembly have been characterised by a set of parameters, geometrical, functional, and technological and so on.

All the parts, the sub-assemblies and the final assembly constituting the press brake were defined as well as their parameters; 3D parametric models of each part were modeled with a $3 \mathrm{D}$ CAD commercial system. The procedures that manage the complete dimensioning of the machine were represented by using the programming language of the development tool. 


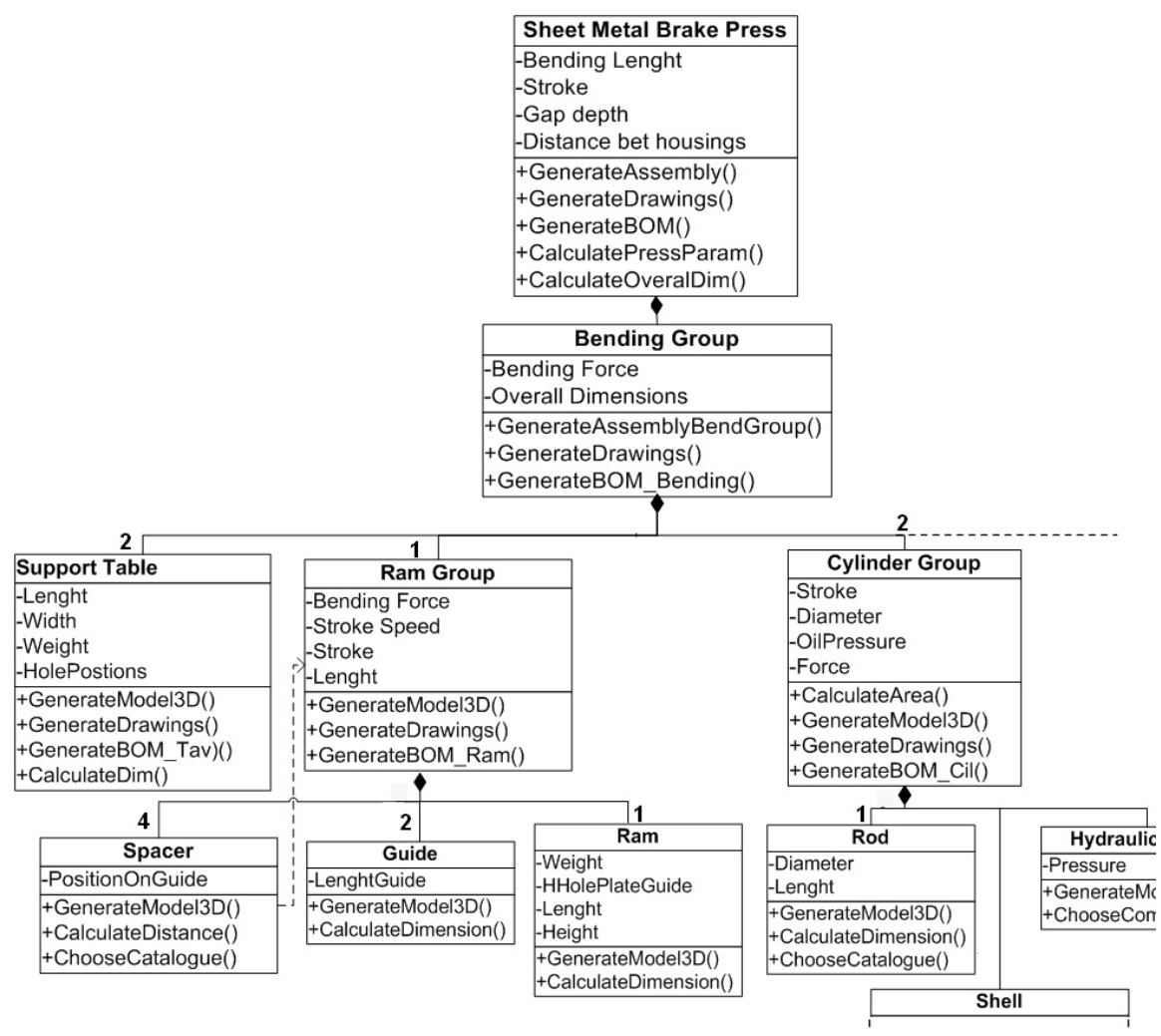

Figure 2 A partial view of the UML static class diagram for the bending group.

Figure 4 portrays the user interface to configure a brake press. The user choices a specific model (in this case PS75, with a maximum bending force of $750 \mathrm{kN}$ ) and, once introduced configuration parameters (bending force, bending length, distance between housing, gap depth, gap height and stroke), the application dimensions automatically all the parts and assemblies, generates 3D models, electronic BOM and 2D drawings. Figure 5 portrays the virtual prototype of a press brake produced by the application, and an example of a 2D drawing.

Summarizing, the results of the application are the correct dimensioning of parts and configuration of the entire product, the 3D CAD models and engineering drawings of each part, the EBOM (Electronic Bill of Materials) and finally cost estimation. In addition, with such an application the product development time, according to customers' requirements, has been dramatically reduced from some days to about 40 minutes. 


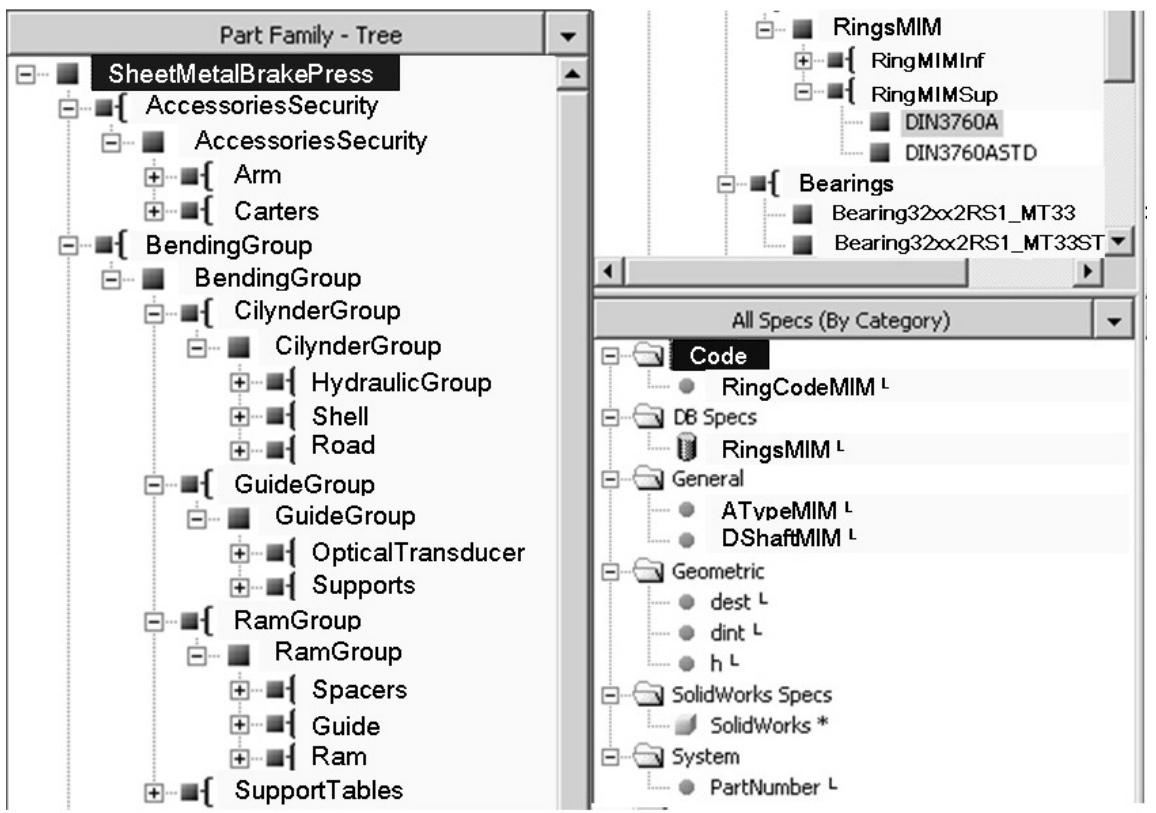

Figure 3 Tree structure implemented within DA application.

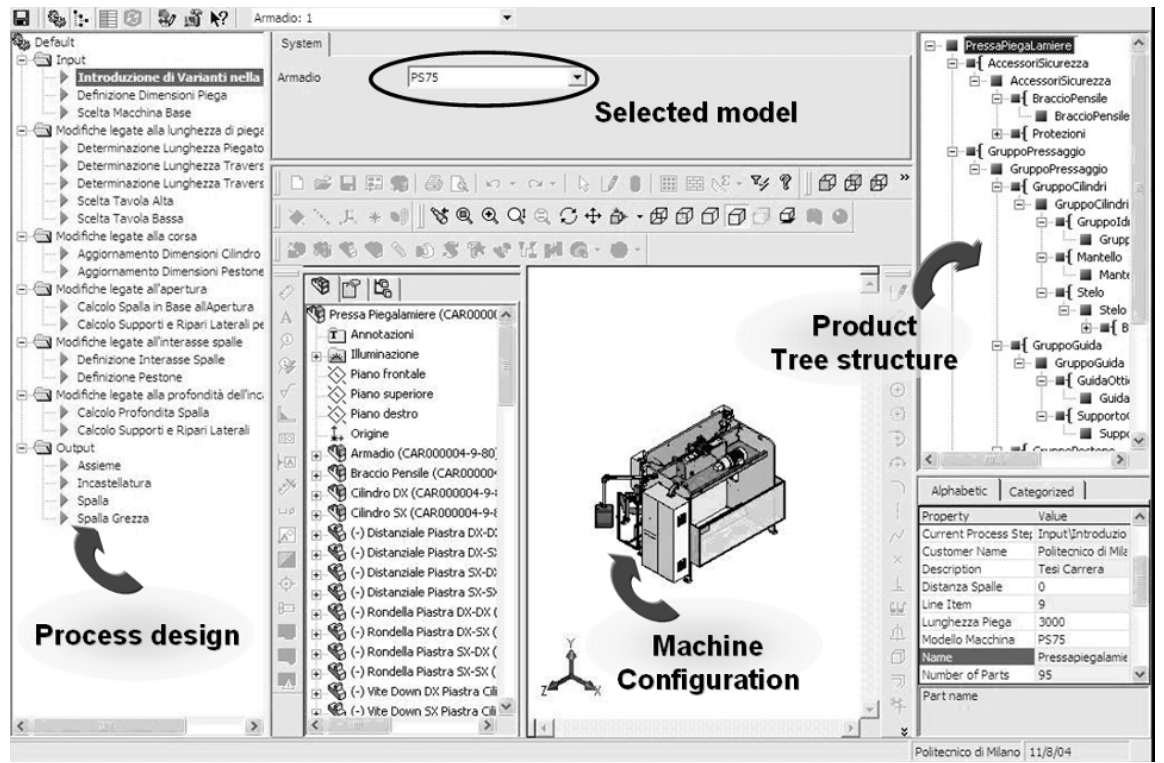

Figure 4 DA application user inteface. 


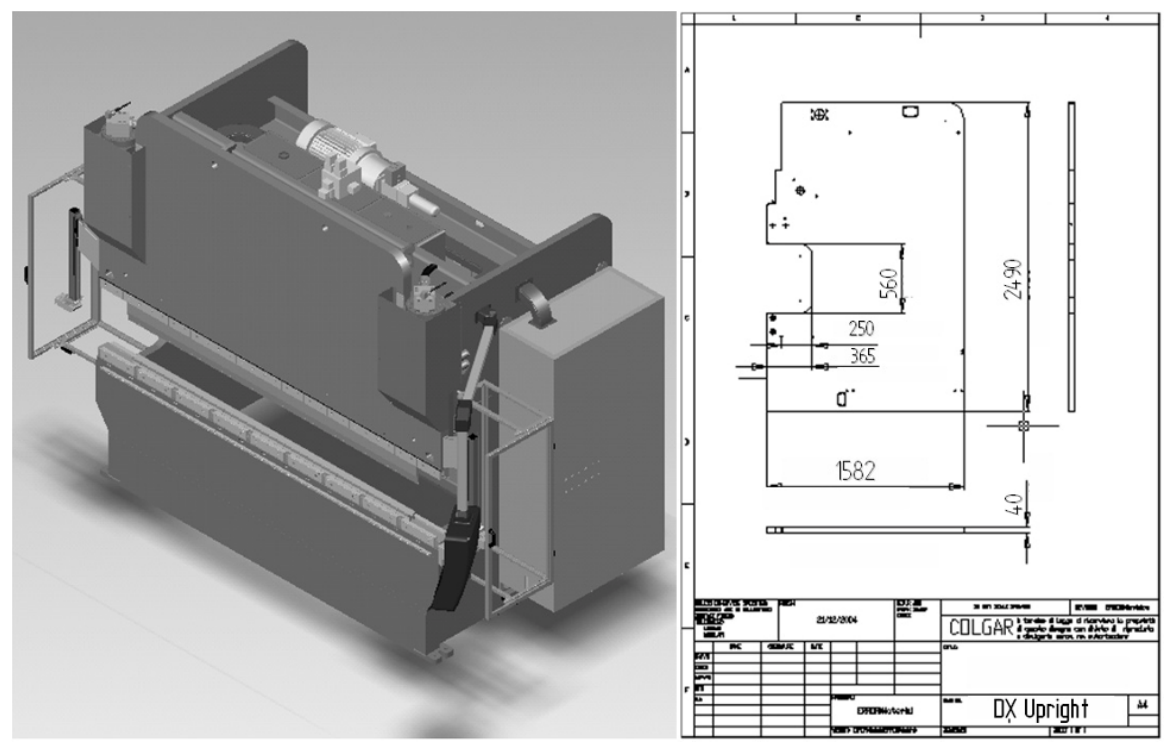

Figure $53 \mathrm{D}$ virtual model of the press and a related 2D drawings.

\section{5. $2^{\text {nd }}$ Study Case: Gear Box}

This case is focused on design process representation and integration between KBE and companies' data repositories for PLM/PDM systems to improve part reuse process in mechanical companies. The process representation requires the software definition of the activities necessary to complete the design of a product, and the development of a DA application in this case, may be a direct mapping of the tasks evidenced in process formalization.

The test case concerns the automatic design of a family of gear box.

As previously, before implementing the application with the KBE kernel, knowledge acquisition and formalization are needed. A deep analysis of the design process currently carried out within the SME has been performed to identify for each activity the objects to be defined, the design tasks, tools, criteria and rules, information sources, etc..

Figure 6 shows the main IDEF0 diagram representing the design process followed within the company. It forecasts four main phases: Define number of shafts, Size gears, Size shafts and Size bearings.

As said, this test case deals also with the integration of the DA application with PLM/PDM system; therefore it has been necessary to define proper strategies to manage the interactions. We defined an approach based on the coding of the parts to solve this problem. The code should summarize functional information on the specific component. This method has some disadvantages due mainly to 
complexity and uniqueness of the code, but at the moment it seems the only reasonable approach. As an example, the following is the code used to define a specific gear (Figure 7).

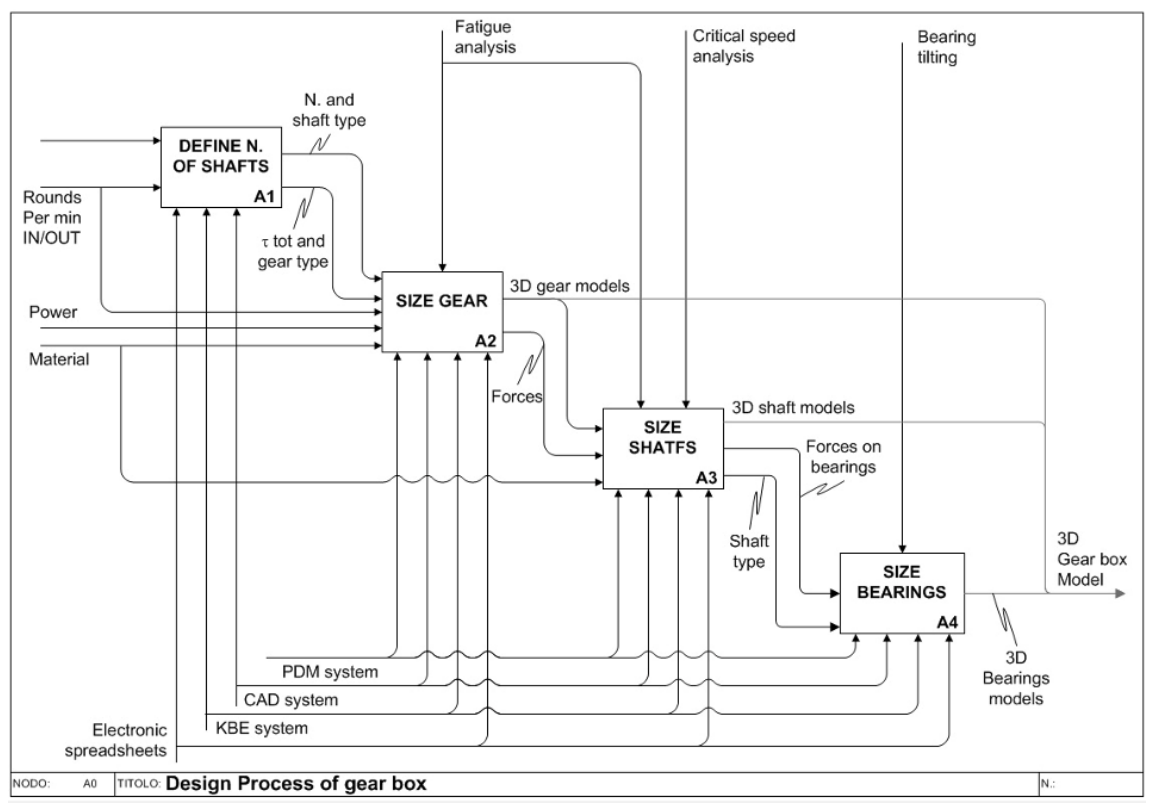

Figure 6 IDEF0 diagram representing the design process for a gear box.

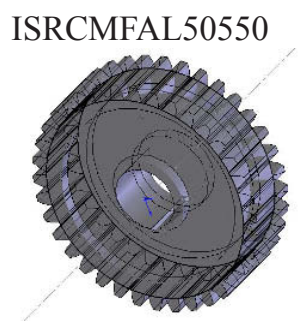

Figure 7 Code for a gear box.

The first four letters specify the type of gear (ISCR, cylindrical with right teeth), the two subsequent letters the fit type (MF), the two final letters the body type (AL), and, then, the teeth number, modulus and pitch diameter.

In such a way, to identify already existing parts, the KBE application can query in an automatic way company's data repositories. Moreover, such an approach makes possible to reuse previously developed specific applications. 
We used as development tool a KBE system process-based. In this case, the KBE kernel of the DA application is a "master" that pilots a lot of "slaves, each of them executing a specific task. "Slaves" can be a spreadsheet, a CAD system, a FE solver, a calculus tool, a PDM/PLM module and so on.

The implemented DA application comprehends several sub-processes, each of them performing a specific design task, e.g., size bearings. These sub-processes properly manage electronic spreadsheet containing all design rules for each system components.

Figure 8 portrays the main window of the user interface where the designer introduces the main design parameters (e.g., transmission power); then the application automatically generates the code associated to the selected type of gear box, and searches for an already existing configuration within the PDM system. If it does not exist, the design procedure starts performing each design step through the execution of corresponding sub-processes and spreadsheets. The application can also proposes a standard solution but the designer can decide to accept it or go on with the definition of a new gear box. Once completed the configuration process, the system generates the 3D model of the final product. Figure 9 portrays two different configurations: one with parallel axis and another with incident axis. The applications developed can also used to train new designers. The possibility to follow gradually the task sequence and the simultaneous control of design parameters and rules allow the junior designers to easily and rapidly acquire the knowledge necessary to work within considered context.

\section{Conclusions}

The paper discusses some aspects related to development of DA application within an industrial context. It focuses the attention of some DA fundamental issues related to the main characteristics of design processes carried out by SMEs.

A methodology, MEDEA, specifically targeted to SME domain, has been presented. It covers all the aspects related to development of a DA application and proposes tools to be utilized in contexts without high-level skills and IT resources. The validation has been performed considering various case studies and the direct involvement of companies' staff more skilled in engineering than in IT technologies. This permitted to verify the effectiveness and the applicability of DA approach in SMEs. Two test cases have been described. The first has been developed applying a more classical way, and using a KBE kernel derived from the traditional approach based on Object-Oriented programming. The latter presents a new approach: the KBE tool utilized permits a direct representation of the design process. In such an application, KBE kernel has been used only to represent all the activities of the design process; the execution of these activities is left to specific slaves, implemented with different tools (CAD, spreadsheet, etc.). 
Finally, we can say that DA is a tool to innovate the design process and not the product itself. Only a superficial analysis may consider an application to automate the design process of a family of products as an obstacle to innovation; but it is the better way to choice, configure and dimension parts and thus a powerful tool to obtain new technical solutions.

\begin{tabular}{|c|c|}
\hline & Automatic Design of a gear box \\
\hline \multirow[t]{13}{*}{ WebPIayer } & \\
\hline & Transmission Power \\
\hline & 55 \\
\hline & N_Revolutions IN \\
\hline & 1500 \\
\hline & N_Revolutions OUT \\
\hline & 250 \\
\hline & Centre Distance \\
\hline & 500 \\
\hline & Select Gear box configuration \\
\hline & Gear box with parallel axes \\
\hline & Default solution \\
\hline & Visualize default solution \\
\hline
\end{tabular}

Figure 8 Application user interface.

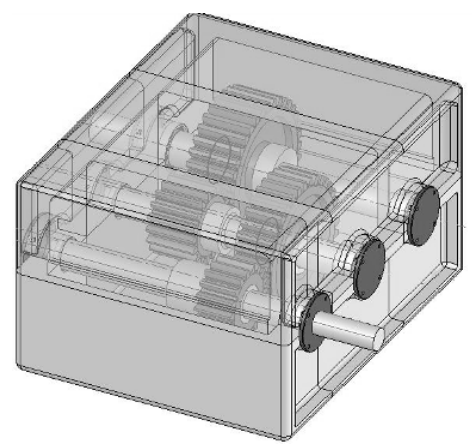

a. Parallel axis

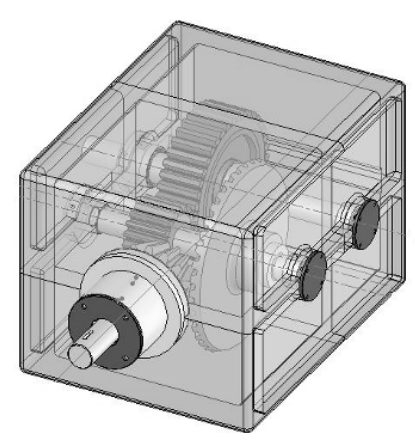

b. Incident axis

Figure 9 Two gear box configurations.

\section{References}

1 Choi J.C, Kim C.: A Compact and Practical CAD/CAM System for the Blanking or Piercing of Irregular Shaped-sheet Metal Products for Progressive Working. J. of Materials Processing Technology, n. 110, pp. 36-46 (2001). 
2 Colombo G., Ferretti D., Cugini U.: How to Represent Design Rules in a Parametric Cad System. In: Proceedings International Symposium on Advanced Geometric Modelling for Engineering Applications, 8-9 November, Berlin, pp. 271-282 (1989).

3 La Rocca, G., Krakers L., van Tooren M.J.L.: Development of an ICAD generative model for blended wing body aircraft design. In: Proceedings 9th Symposium on multidisciplinary analysis and optimization, AIAA2002-5447 (2002).

4 Craig B., Chapman M.P.: The Application of a Knowledge Based Engineering Approach to the Rapid Design and Analysis of an Automotive Structure. Advances in Engineering Software, vol. 32, pp. 903-912, Elsevier (2001).

5 Stokes M.: Managing Engineering Knowledge - MOKA: Methodology for Knowledge Based Engineering Applications. Professional Engineering Publishing, London and Bury St Edmunds, UK (2001).

6 Pinfold M., Chapman C., Preston S.: Knowledge Application Methodology For Knowledge Based Product Development Automation (KAM) (2006).

7 Lovett P.J., Ingram A., Bancroft C.N.: Knowledge-based engineering for SMEs - a methodology. J. of Materials Processing Technology, pp. 384-389, Elsevier (2000).

8 Klein R.: Knowledge Modeling in Design - the MOKA framework. In: Proceedings of the International AI in Design Conference, JS Gero (ed), Kluwer, Worcester, MA (2000).

9 Forster J., Arana I., Fothergill P.: Re-Design Knowledge Representation with DEKLARE in: A domain model oriented, industrial KA framework applied to VT. Available at http://citeseer.ist.psu.edu/351655.html.

10 Harper A.P.: Knowledge-based engineering. Engineering Designer, vol. 25, n. 1, pp. 29-32 (1999).

11 Susca L., Mandorli F., Rizzi C., Cugini U.: Racing car design using knowledge aided engineering. AI EDAM, vol. 14, n. 3, pp. 235-249 (2000).

12 Shah R.K., Sekulic D.P.: Fundamentals of Heat Exchanger Design, John Wiley \& Sons (2002).

13 Preston S., Chapman C., Pinfold M., Smith G.: Knowledge acquisition for knowledge-based engineering systems. Int. J. Information Technology and Management, vol. 4, n.1 (2005).

14 Gorti S.R., Gupta A., Kim G.J., Sriram R.D., Wong A.: An object-oriented representation for product and design processes. Computer-Aided Design, vol. 40 n. 7, Springer, pp. 489-501 (1998).

15 Colombo G., Cugini U., Pugliese D., Pulli M.: Levels of knowledge representation for Product Design. In: Proceeding International Conference on Product Lifecycle Management, Lyon, France, 11-13 July 2005 (2005).

16 Colombo G., Pugliese D.: The Role of Knowledge Management in Product Lifecycle. Innovation in Life Cycle Engineering and Sustainable Development, D. Brissaud, S. Tichkiewitch and P. Zwolinski (eds), Springer, pp. 397-406 (2006).

17 Sheer W., Abolhassan F., Jost W., Kirchmer M.: Business process excellence - ARIS in practice, Springer, Berlin (2002).

18 Eriksson H., Penker M.: Business modeling with UML. John Wiley and Sons (2000).

19 Sainter P., Oldham K., Larkin A., Murton A., Brimble R.: Product Knowledge Management within Knowledge Based Engineering Systems. In: Proceedings of ASME 2000 Design Engineering Technical Conference and Computers and Information in Engineering Conference, Baltimore, Maryland (2000).

20 Cheung P.K., Chau P.Y.K., Au, A.K.K.: Does Knowledge Reuse Make a Creative Person More Creative? In Proceeding The Ninth Pacific Asia Conference on Information Systems (PACIS-2005), Bangkok, Thailand (2005). 